\title{
Changes in D-dimer after initiation of antiretroviral therapy in adults living with HIV in Kenya
}

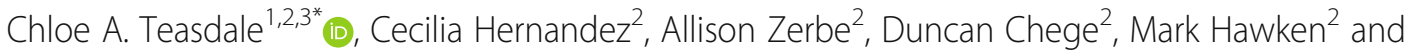
Wafaa M. El-Sadr ${ }^{2,3}$

\begin{abstract}
Background: Increased coagulation biomarkers are associated with poor outcomes among people living with HIV (PLHIV). There are few data available from African cohorts demonstrating the effect of antiretroviral therapy (ART) on coagulation biomarkers.
\end{abstract}

Methods: From March 2014 to October 2014, ART-naive PLHIV initiating non-nucleoside reverse transcriptase inhibitorbased ART were recruited from seven clinics in western Kenya and followed for up to 12 months. Demographics, clinical history and blood specimens were collected. Logistic regression models adjusted for intrasite clustering examined associations between HIV viral load and D-Dimer at baseline. Mixed linear effects models were used to estimate mean change from baseline to 6 months overall, and by baseline viral load, sex and TB status at enrollment. Mean change in D-dimer at 6 months is reported on the log 10 scale and as percentage change from baseline.

Results: Among 611 PLHIV enrolled, 66\% were female, median age was 34 years (interquartile range (IQR) 29-43 years), 31 (5\%) participants had tuberculosis and median viral load was 113,500 copies/mL (IQR: 23,600-399,000). At baseline, 311 (50.9\%) PLHIV had elevated D-dimer ( $\geq 500 \mathrm{ng} / \mathrm{mL}$ ) and median D-dimer was $516.4 \mathrm{ng} / \mathrm{mL}$ (IQR: 302.7-926.6) (log baseline D-dimer: 2.7, IQR: 2.5-3.0). Higher baseline D-dimer was significantly associated with higher viral load ( $p<$ $0.0001)$, female sex $(p=0.02)$ and tuberculosis $(p=0.02)$. After 6 months on ART, $518(84.8 \%)$ PLHIV had achieved viral load < 1000 copies/mL and median D-dimer was 390.0 (IQR: 236.6-656.9) (log D-dimer: 2.6, IQR: 2.4-2.8). Mean change in log D-dimer from baseline to 6 months was $-0.12(95 \% \mathrm{Cl}-0.15,-0.09)(p<0.0001)$ indicating at $31.3 \%$ decline $(95 \% \mathrm{Cl}-40.0,-23.0)$ in D-dimer levels over the first 6 months on ART. D-dimer decline after ART initiation was significantly greater among PLHIV with tuberculosis at treatment initiation $(-172.1,95 \% \mathrm{Cl}-259.0,-106.3 ; p<0.0001)$ and those with log viral load $>6.0$ copies $/ \mathrm{mL}(-91.1,95 \% \mathrm{Cl}-136.7,-54.2 ; p<0.01)$.

Conclusions: In this large Kenyan cohort of PLHIV, women, those with tuberculosis and higher viral load had elevated baseline D-dimer. ART initiation and viral load suppression among ART-naïve PLHIV in Kenya were associated with significant decrease in D-dimer at 6 months in this large African cohort.

Keywords: Coagulation, D-dimer, ART initiation, Tuberculosis, Women

\footnotetext{
* Correspondence: hloe.teasdale@sph.cuny.edu

1Department of Epidemiology and Biostatistics, CUNY Graduate School of

Public Health and Health Policy, 55 W125th Street, Room 543, New York, NY 10027, USA

${ }^{2}$ ICAP at Columbia University, Mailman School of Public Health, Columbia University, 22 W168th Street, New York, NY 10032, USA

Full list of author information is available at the end of the article
}

(C) The Author(s). 2020 Open Access This article is licensed under a Creative Commons Attribution 4.0 International License, which permits use, sharing, adaptation, distribution and reproduction in any medium or format, as long as you give appropriate credit to the original author(s) and the source, provide a link to the Creative Commons licence, and indicate if changes were made. The images or other third party material in this article are included in the article's Creative Commons licence, unless indicated otherwise in a credit line to the material. If material is not included in the article's Creative Commons licence and your intended use is not permitted by statutory regulation or exceeds the permitted use, you will need to obtain permission directly from the copyright holder. To view a copy of this licence, visit http://creativecommons.org/licenses/by/4.0/ The Creative Commons Public Domain Dedication waiver (http://creativecommons.org/publicdomain/zero/1.0/) applies to the data made available in this article, unless otherwise stated in a credit line to the data. 


\section{Background}

Increased levels of D-dimer, a marker of hyper coagulation, have been shown to be a strong predictor of morbidity and mortality among people living with HIV (PLHIV) both prior to and after initiation of antiretroviral therapy (ART) [1-3]. The Strategies for Management of Antiretroviral Therapy (SMART) study provided important insights into the correlation between coagulation and inflammatory markers in PLHIV and increased mortality. The study found that D-dimer and interleukin-6 (IL-6) were the strongest predictors of all-cause mortality among a population of relatively healthy PLHIV [4]. Other studies have also shown that elevated D-dimer levels in PLHIV both before and after ART initiation are correlated with greater risk of nonAIDS events and mortality in resource-rich [2, 5] and resource-limited settings among populations with advanced HIV disease [3, 6, 7]. PLHIV, including those on ART, appear to be at higher risk for venous thrombotic events $[8,9]$ and pro-coagulant states $[10,11]$ which have been linked to continued immunodeficiency and low level viral replication even after treatment initiation $[4,11]$.

While long-term ART, particularly with use of specific antiretroviral drugs, has been associated with some metabolic complications, including hyperlipidemia and myocardial infarction [12, 13], ART also has beneficial effects in decreasing inflammatory and coagulation biomarkers. Studies have shown declines in D-dimer levels following treatment initiation, and have further demonstrated that delayed ART, poor treatment adherence and interruption of ART may contribute to elevated coagulation markers and increased risk of death [4, 14-16]. Nonetheless, compared to HIV-negative persons, higher D-dimer levels have been shown to persist even in PLHIV who maintain viral suppression on long-term ART [14, 17]. Few studies have examined change in D-dimer resulting from ART initiation including examination of pre-treatment measures, and most research in this area has been conducted in resourcerich settings. In this analysis, we present an investigation of changes in D-dimer levels after ART initiation among a large cohort of adult PLHIV in Kenya.

\section{Methods}

The Antiretroviral Therapy and Inflammatory and Coagulation Biomarkers (ARTIC) prospective cohort study was designed to examine the relationship between ART use and changes in biomarkers among adult ART-naïve PLHIV initiating treatment in Kenya (NCT02027480). Study participants included male and female PLHIV aged 18 years and older who were recruited from seven health facilities in Nyanza region of Kenya from March through October 2014. All participants were eligible for
ART based on Kenyan national guidelines which changed over the course of the study from CD4+ cell count $(\mathrm{CD} 4+)<350$ cells $/ \mathrm{mm}^{3}$ or World Health Organization (WHO) stage 3 or 4 to CD $4+<500$ cells $/ \mathrm{mm}^{3}$ regardless of WHO stage (as of July 2014) [18]. The recommended first line treatment regimen in Kenya at the time of the study was Tenofovir (TDF) + Lamivudine (3TC) + Efavirenz (EFV) [18]. Women who were currently pregnant were excluded. Kenyan national guidelines called for immediate initiation of tuberculosis (TB) treatment with ART initiation to begin after TB treatment was tolerated, at least within the first 2-8 weeks after start of TB treatment. The preferred ART regimen for those on TB treatment was TDF +3 TC + EFV [18]. Participants who were diagnosed with $\mathrm{TB}$ after the enrollment visit were analyzed according to their TB status at enrollment. The ARTIC study was approved by the Kenya Medical Research Institute (KEMRI) and the Columbia University Medical Center (CUMC) Institutional Review Boards (IRB).

Consented participants attended an enrollment visit followed by visits at two, six and 12 months. The enrollment visit included collection of demographic, medical history, physical examinations including height and weight measurements, and collection of blood and urine samples. Participants received routine care at participating health facilities per national guidelines including initiation of non-nucleoside reverse transcriptase inhibitor (NNRTI)-based ART regimens. At all study follow-up visits, clinical data were abstracted from medical charts including CD4+ cell count and ART regimens. At six and 12 months, physical examinations included measurement of weight and blood pressure, and collection of blood samples to assess biomarker and HIV viral load over time. Blood specimens were collected by venipuncture using vacutainer tubes. Within $12 \mathrm{~h}$ of collection, specimens were transported to the KEMRI/CDC laboratory where they were stored at $-80 \mathrm{C}$ until thawed for batched biomarker evaluation at the University of Nairobi Institute of Tropical and Infectious Diseases Laboratory (Nairobi, Kenya). We report measures of HIV viral load copies/mL (Xpert ${ }^{\oplus}$ HIV-1 Viral Load, Cepheid AB, Solna, Sweden) and D-dimer in nanograms (ng)/mL (Nano-Check ${ }^{\mathrm{Tm}}$ kits, Nano-Ditech, New Jersey, USA). The lower limits of detection was 40 copies/mL for viral load and $200 \mathrm{ng} / \mathrm{mL}$ for D-dimer.

In this analysis, we describe study population characteristics at enrollment by D-dimer level including sex, age, CD4+ cell count, viral load level, body mass index (BMI) and TB status. D-dimer was dichotomized as elevated $(\geq 500 \mathrm{ng} / \mathrm{mL})$ and normal $/$ low $(<500 \mathrm{ng} / \mathrm{mL})$ based on standard (non-age adjusted) cutoffs used to identify activated coagulation in patients [19]. BMI was defined as individual's weight divided by the square of 
their height $\left(\mathrm{kg} / \mathrm{m}^{2}\right)$ and was categorized in accordance with the WHO guidelines [20]. TB diagnosis at the time of study enrollment was ascertained from clinical records. Chi-square tests for proportions and Wilcoxon tests for medians were used to assess the unadjusted association of D-dimer at baseline with participant demographic and clinical characteristics including sex, age, CD4+ cell count, viral load, BMI and TB at study enrollment. We also examined the correlation between age and baseline D-dimer as a continuous variable using Pearson correlation coefficients. We report unadjusted median D-dimer levels at baseline and 6 months after ART initiation overall and according to pre-treatment viral load, sex and TB status at enrollment. D-dimer was not normally distributed and was evaluated in models on the $\log 10$ scale. To estimate mean change in $\log \mathrm{D}$ dimer values from baseline to 6 months, analysis of variance (ANOVA) models (random effects linear models) adjusted for baseline D-dimer were used. We also estimated mean log D-Dimer change in participants according to baseline viral load, sex and TB status at enrollment; separate bivariable models (adjusted for baseline D-Dimer) were run to estimate mean D-Dimer change by levels of the baseline characteristic (not adjusted for other variables). Mean change estimates were back transformed to the original D-dimer scale and presented as percent change from baseline. For examination of TB at enrollment (identified through routine care), we conducted an additional analysis including participants diagnosed with TB between the baseline and 2 month study visit; however these analyses did not yield results differing from the analysis with only those diagnosed at the time of study enrollment and are not presented.

\section{Results}

Among 685 ART-naïve adult PLHIV enrolled in the ARTIC study, 611 (89.2\%) were included in this analysis. Forty-nine (7.2\%) participants were lost to follow-up after enrollment and another $25(3.6 \%)$ were missing Ddimer measures at either baseline or 6 months. As shown in Table 1, among the included participants, half (50.9\%) had high D-dimer levels ( $\geq 500)$ at study enrollment and median D-dimer overall was 516.4 (interquartile range (IQR): 302.7-926.6). Most participants were female (65.5\%) and median age was 34 years (IQR: 2943). At baseline median CD4+ count was 326 cells $/ \mathrm{mm}^{3}$ (IQR 194-442) and median viral load was 113,500 copies/mL (IQR 23,600-399,000); half (50.4\%) of all participants had a viral load $>100,000$ copies $/ \mathrm{mL}$. Among 31 (5.0\%) participants with $\mathrm{TB}$ at study enrollment, 19 (61.3\%) were male, 29 (93.5\%) had pulmonary TB (16 of whom were smear positive and 13 were smear negative) and two (6.5\%) had extra-pulmonary TB. In addition, five participants were diagnosed with $\mathrm{TB}$ between the enrollment and the six-month visit (four were pulmonary smear negative and one was extra-pulmonary); four between the enrollment and 2 month visit and one additional participant between the 2 and 6 month visits. Most participants (69.5\%) had normal BMI (18.5-24.9). The proportion of women with high D-dimer levels at baseline was more than double that for men $(p=0.02)$. Elevated D-dimer levels were also associated with higher viral load, 56.6\% of PLHIV with elevated D-dimer had baseline viral load $>100,000$ copies $/ \mathrm{mL}$ compared to $44.0 \%$ of those with normal/low D-dimer $(p=0.01)$. PLHIV with TB disease at baseline were also more likely to have elevated D-dimer $(p=0.02)$. BMI and CD4+ at study enrollment were not associated with elevated baseline D-dimer. Age was also not associated with elevated baseline D-dimer when examined as a dichotomous variable $(p=0.60)$ (Table 1$)$, nor as a continuous variable (correlation coefficient $(\mathrm{r})=0.03, p=0.52$ ).

At 6 months after starting ART, 518 (84.8\%) PLHIV had achieved a viral load $<1000$ copies $/ \mathrm{mL}(5.7 \%$ missing viral load at 6 months). Median D-dimer at 6 months after ART initiation was 390.0 (IQR: 236.6-656.9) (log 2.6, IQR: 2.4-2.8) (Table 2). Mean log change for all participants from baseline to 6 months was $-0.12(95 \% \mathrm{CI}$ $-0.15,-0.09)$. Median D-dimer at baseline among PLHIV with $\log$ viral load $>6.0 / \mathrm{mL}(819.8 \mathrm{ng} / \mathrm{mL})$ and mean change at 6 months $(-0.28(95 \% \mathrm{CI}-0.37,-0.19)$ was significantly higher compared to those with lower baseline viral load $(p<0.001)$ (Table 2). PLHIV with TB at enrollment had the highest baseline D-dimer (median 1230.3, IQR: 465.1-2586.3) and mean change in log Ddimer at 6 months in these participants was significantly higher than in PLHIV without TB $(-0.43,95 \% \mathrm{CI}-0.56$, $-0.31, p<0.0001)$. Although women had higher median D-dimer at baseline compared to men (566.1 vs. 448.0$)$, there was no significant difference in mean change from baseline to 6 months in $\log \mathrm{D}$-dimer by sex $(p=0.14)$ (Table 2). Overall, there was a $31.3 \%$ decline in D-dimer from baseline to 6 months after ART initiation (95\%CI -40.0, - 23.0) (Fig. 1). Among PLHIV starting ART with $\log$ viral load $>6.0$ copies $/ \mathrm{mL}$, there was a $91.1 \%(95 \% \mathrm{CI}$ -136.7, - 54.2) decrease at 6 months on ART, while those with baseline log viral load $<4.0$, had a mean decrease of $19.1 \%$ (95\%CI $-39.0,-2.1)$. PLHIV with TB at ART initiation saw the largest percent decline in Ddimer levels, $172.1 \%$ (95\%CI -259.0, - 106.3).

\section{Discussion}

In this cohort of 611 ART-naive, treatment-eligible Kenyan adults, almost half had elevated pre-ART Ddimer levels $(\geq 500 \mathrm{ng} / \mathrm{mL})$ indicating increased risk for morbidity and mortality. Women, PLHIV with higher viral load and those with TB at ART initiation were most likely to have elevated pre-treatment D-dimer. At 
Table 1 Characteristics at study enrollment of ART-naïve treatment eligible adults ( $\geq 18$ years) living with HIV in Kenya and starting ART in 2014 according to enrollment D-dimer level, normal/low vs. high ( $N=611)$

\begin{tabular}{|c|c|c|c|c|c|c|c|}
\hline & \multicolumn{2}{|c|}{ Total } & \multicolumn{2}{|c|}{$\begin{array}{l}\text { Normal/low D-dimer } \\
(<500 \mathrm{ng} / \mathrm{mL})\end{array}$} & \multicolumn{2}{|c|}{$\begin{array}{l}\text { High D-dimer } \\
(\geq 500 \mathrm{ng} / \mathrm{mL})\end{array}$} & \multirow{3}{*}{$p$-value } \\
\hline & $\mathrm{N}$ & $\%$ & $\mathrm{~N}$ & $\%$ & $\mathrm{~N}$ & $\%$ & \\
\hline & 611 & 100.0 & 300 & 49.1 & 311 & 50.9 & \\
\hline Enrollment D-dimer, median (IQR) & \multicolumn{2}{|c|}{$516.4(302.7-926.6)$} & \multicolumn{2}{|c|}{$301.7(199.0-397-6)$} & \multicolumn{2}{|c|}{$912.8(690.2-1446.2)$} & $<0.0001$ \\
\hline \multicolumn{8}{|l|}{ Sex } \\
\hline Male & 211 & 34.5 & 117 & 39.0 & 94 & 30.2 & 0.02 \\
\hline Female & 400 & 65.5 & 183 & 61.0 & 217 & 69.8 & \\
\hline Age (years), median (IQR) & \multicolumn{2}{|c|}{$34(29-43)$} & \multicolumn{2}{|c|}{$34(29-43)$} & \multicolumn{2}{|c|}{$34(29-43)$} & 0.86 \\
\hline$<25$ & 52 & 8.5 & 29 & 9.7 & 23 & 7.4 & 0.60 \\
\hline $25-40$ & 384 & 62.9 & 186 & 62.0 & 198 & 63.7 & \\
\hline$>40$ & 175 & 28.6 & 85 & 28.3 & 90 & 28.9 & \\
\hline CD4+ cells/mm3, median (IQR) & \multicolumn{2}{|c|}{$326(194-442)$} & \multicolumn{2}{|c|}{$335(221-451)$} & \multicolumn{2}{|c|}{$319(177-435)$} & 0.17 \\
\hline$>500$ & 78 & 12.8 & 39 & 13.0 & 39 & 12.5 & 0.42 \\
\hline $350-500$ & 186 & 30.4 & 98 & 32.7 & 88 & 28.3 & \\
\hline $200-349$ & 190 & 31.1 & 95 & 31.7 & 95 & 30.6 & \\
\hline$<200$ & 156 & 25.5 & 68 & 22.7 & 88 & 28.3 & \\
\hline Missing & 1 & 0.2 & 0 & 0.0 & 1 & 0.3 & \\
\hline Viral load copies/mL, median (IQR) & \multicolumn{2}{|c|}{$113,500(23,600-399,000)$} & \multicolumn{2}{|c|}{$82,200(15,800-294,000)$} & \multicolumn{2}{|c|}{$159,000(33,400-501,000)$} & 0.0001 \\
\hline$<1000(<\log 3.0)$ & 30 & 4.9 & 18 & 6.0 & 12 & 3.9 & 0.01 \\
\hline 1000-9999 (log 3.0-3.9) & 57 & 9.3 & 34 & 11.3 & 23 & 7.4 & \\
\hline 10,000-99,999 $(\log 4.0-<4.9)$ & 201 & 32.9 & 107 & 35.7 & 94 & 30.2 & \\
\hline $100,000-1000,000(\log 5.0-5.9)$ & 255 & 41.7 & 116 & 38.7 & 139 & 44.7 & \\
\hline$>1000,000(>\log 6.0)$ & 53 & 8.7 & 16 & 5.3 & 37 & 11.9 & \\
\hline Missing & 15 & 2.5 & 9 & 3.0 & 6 & 1.9 & \\
\hline BMI, median (IQR) & \multicolumn{2}{|c|}{$20.9(18.9-22.9)$} & \multicolumn{2}{|c|}{$21.1(19.1-22.9)$} & \multicolumn{2}{|c|}{$20.5(18.6-23.0)$} & 0.21 \\
\hline Underweight (<18.5) & 114 & 18.7 & 48 & 16.0 & 66 & 21.2 & 0.17 \\
\hline Normal (18.5-24.9) & 425 & 69.6 & 219 & 73.0 & 206 & 66.2 & \\
\hline Overweight \& Obese ( $\geq 25$ ) & 72 & 11.8 & 33 & 11.0 & 39 & 12.5 & \\
\hline Current TB at study enrollment & 31 & 5.1 & 9 & 3.0 & 22 & 7.1 & 0.02 \\
\hline
\end{tabular}

6 month visit after starting ART, D-dimer levels decreased, with the median falling to below the threshold indicative of elevated coagulation. The most significant declines were observed among those with highest pretreatment levels, including participants with high pretreatment viral load and those with co-existing TB. To our knowledge, this is largest cohort report of changes in this critical coagulation marker after ART initiation from a sub-Saharan African country and our findings provide important information about the benefits of treatment initiation, particularly for PLHIV with advanced disease.

At study enrollment, among all treatment eligible PLHIV in Kenya with median viral load of roughly 100, 000 copies $/ \mathrm{mL}$, we observed a median D-dimer of 516 $\mathrm{ng} / \mathrm{mL}$ which is above the clinical cutoff of $500 \mathrm{ng} / \mathrm{mL}$.
Our findings showed somewhat lower baseline levels than previous studies of untreated African cohorts, including a study of women living with HIV in Rwanda [21] and another of South African PLHIV with advanced disease [3]. In our cohort, women, PLHIV with high viral load and those with TB had higher pre-treatment Ddimer levels. Sex differences in coagulation makers have been previously documented in HIV-negative populations and in PLHIV enrolled in clinical trials conducted in high resource settings [22-24]. Similar to our results, a study conducted in South Africa and Uganda also found higher pre-treatment D-dimer levels in women compared to men [25]. Previous studies in PLHIV have shown an association between advanced disease and coagulation markers [1, 3, 22, 25], including D-dimer, however we believe this to be one of the largest African 
Table 2 Median biomarker values at baseline and 6 months and mean change from baseline to 6 months among adults ( $\geq 18$ years) starting ART in Kenya 2014 ( $N=611)$

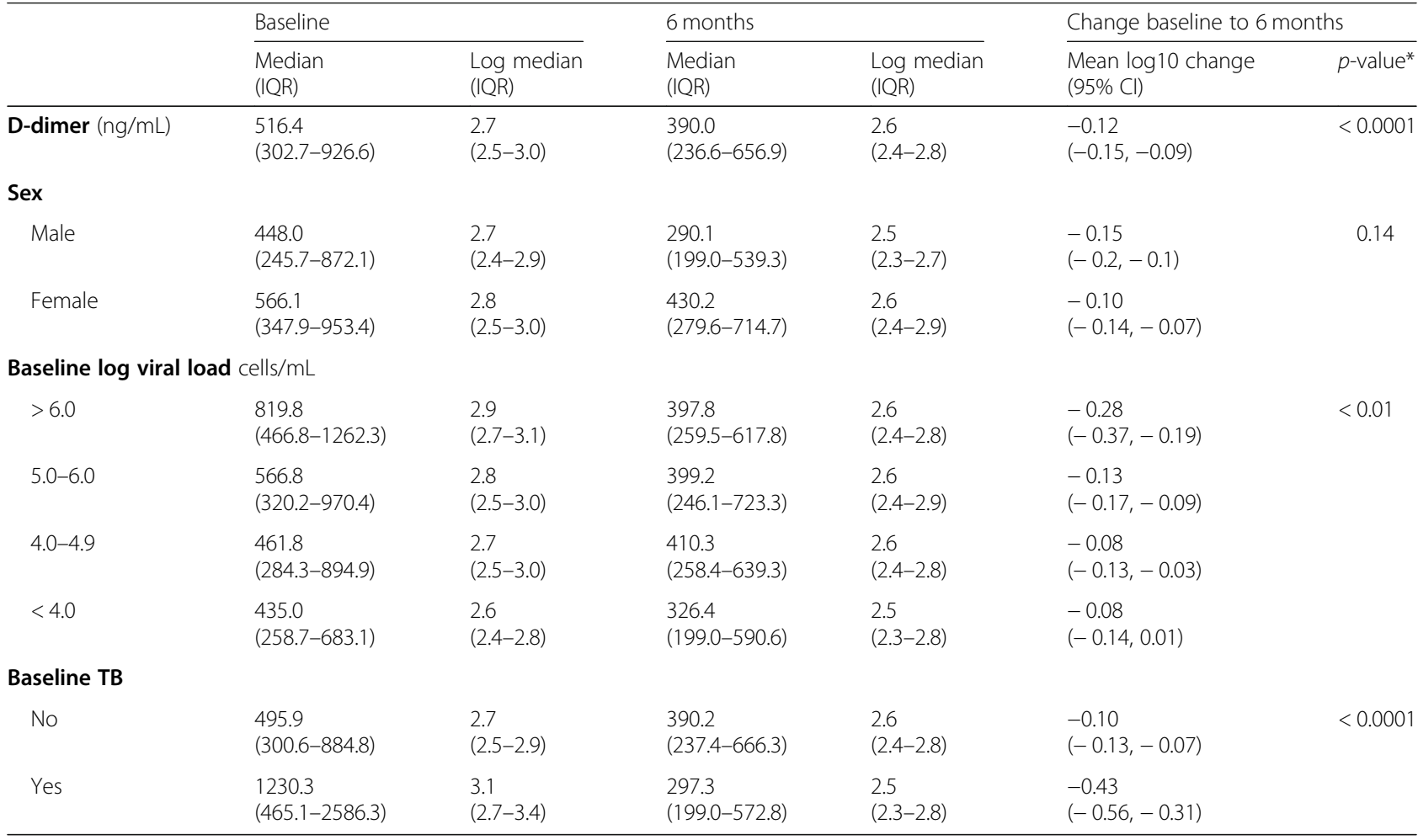

* $p$-values from Type 3 Tests of Fixed Effects

cohorts in which pre-treatment findings have been described. There are few previous reports of the relationship between TB and D-dimer in PLHIV however TB disease has been shown to induce a hypercoagulable state $[26,27]$ and a study of 98 PLHIV in Gabon also reported higher pre-ART D-dimer levels among those with concurrent $\mathrm{TB}$ infection compared to those without [28].

We report significant reduction in D-dimer levels over the first 6 month after ART initiation in Kenyan PLHIV, particularly among those with higher viral load and TB at enrollment. Overall, median D-dimer level at 6 months on treatment was $390 \mathrm{ng} / \mathrm{mL}$ which is below the clinical cutoff of $500 \mathrm{ng} / \mathrm{mL}$ and there was a $31 \%$ decline overall in D-dimer levels after treatment start. PLHIV starting ART with higher viral load and those with TB saw the largest declines in D-dimer. Our results are unique as few studies have evaluated change in coagulation markers after ART initiation among PLHIV coinfected with TB. While we had only a small number of participants with TB at enrollment $(N=31)$, in this cohort, PLHIV with TB had significantly higher pre-ART D-dimer levels compared to those without TB and experienced a $172 \%$ decline in $\mathrm{D}$-dimer, with median $\mathrm{D}$ dimer levels at 6 months within clinically normal values. Our findings are consistent with the previously cited study from Gabon which also found significant decreases in D-dimer among 19 PLHIV with TB after ART initiation [28]. These findings demonstrate the important benefits of treatment initiation for PLHIV particularly those with advanced disease and comorbidities.

While the impact of treatment with antiretroviral medications on levels of coagulation markers in PLHIV has been shown in clinical trials, there are limited findings from real world cohorts, particularly from African settings. The SMART trial found that among participants who achieved viral load $<400$ copies $/ \mathrm{mL}$, at 6 months on ART median decline in D-dimer level was $0.10 \mu \mathrm{g} / \mathrm{mL}$ (IQR: $-0.31-0.00$ ) or a $51 \%$ reduction [15]. The Monitoring of Early Adherence (META) cohort study, conducted in South Africa and Uganda, also showed significant reductions in D-dimer at 12 months after ART initiation among 438 PLHIV [25]. While we examined D-dimer change over the first 6 months on ART in a cohort of PLHIV with more advanced disease than these previous studies, our results are consistent. Our estimate of mean change in the first 6 months after treatment initiation is also similar to a study of 100 PLHIV from South Africa with advanced disease which reported significant reductions in D-dimer at 6 months after ART initiation $(-0.12, p<0.0001)$ [3]. In a cohort of Rwandan women living with HIV, significant 


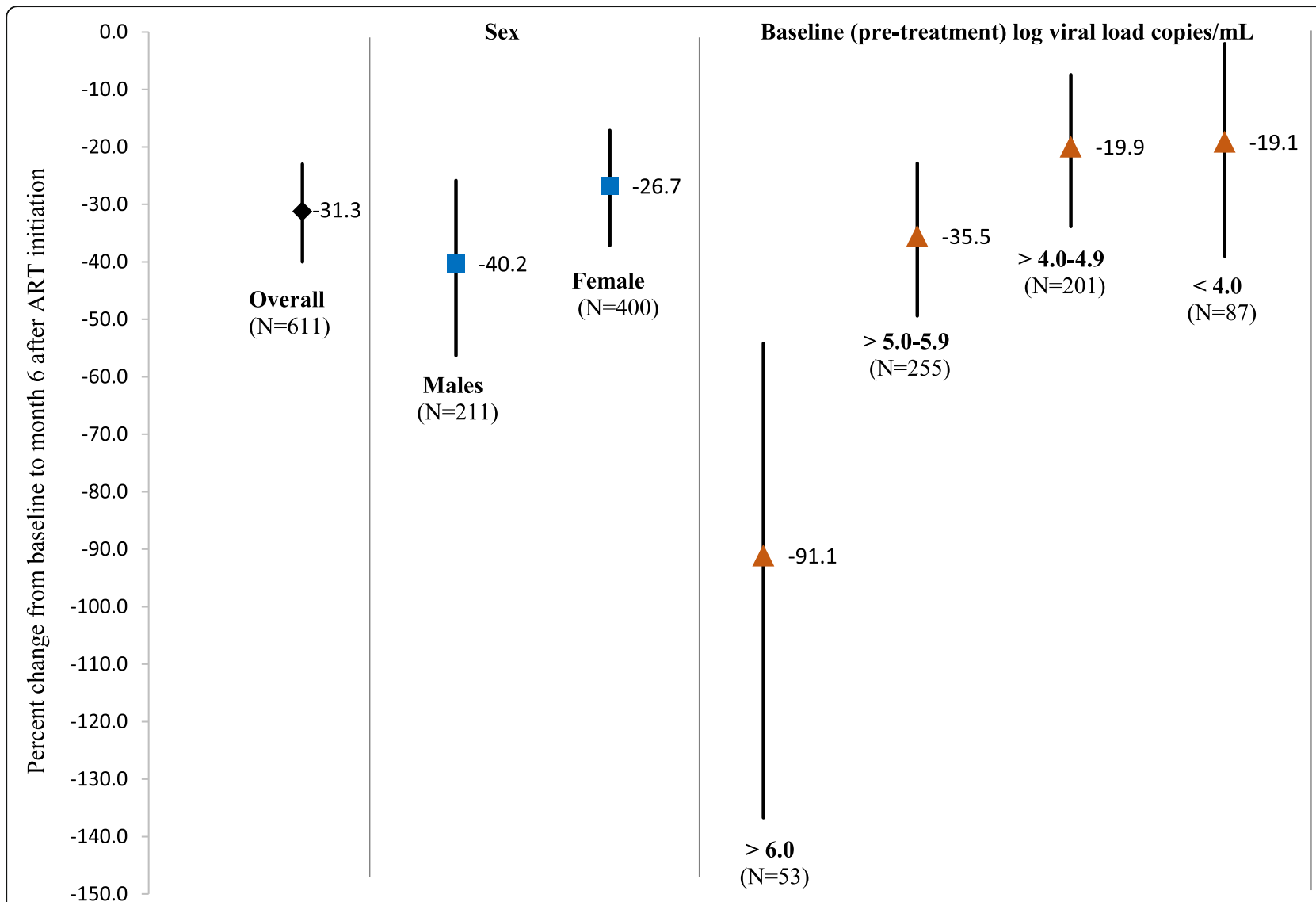

*excludes percent change estaimtes by TB status at enrollment due to scale

Fig. 1 Mean percent change $(95 \% \mathrm{Cl})$ in D-dimer levels from baseline to 6 months after ART initiation among adults ( $\geq 18$ years) starting ART in Kenya 2014 overall, and by sex and baseline log viral load $(N=611)^{*}$

reductions in D-dimer levels were observed at two years on ART demonstrating the continued salutary effect of treatment initiation [21]. The decrease in D-dimer levels as a result of treatment can be anticipated to result in favorable outcomes in terms of thrombotic complications and survival.

There are several important strengths of this analysis. As noted, our findings are unique based on the size and setting of the cohort, as well as the measurement of $\mathrm{D}$ dimer and viral load over the first 6 months of treatment. The large size of this African cohort is important given that the majority of PLHIV receive care in similar settings and the fact that few prior studies have focused on coagulation markers in this population. Our findings demonstrating the effect of ART initiation on D-dimer levels are highly relevant for this population and underscore the importance of treatment initiation to improve long term health outcomes for PLHIV. Our study is also novel with regard to our description of D-Dimer in PLIHV with TB disease; as noted, very few previous studies have examined D-dimer in PLHIV with TB despite it being the leading cause of death among PLHIV [29]. There are also limitations to our analysis including the relatively short duration of follow-up which did not allow us to examine the durability of the reductions we observed in D-dimer. The sample size also limited our ability to examine associations between baseline and change in D-dimer levels with morbidity and mortality outcomes over time. We did not have a comparison group with which to compare our data such as PLHIV starting ART with higher CD4 counts or persons not living with HIV. Our study was conducted prior to the introduction of 'treat all' guidelines in Kenya and during a time when majority of patients initiated NNRTI-based regimens. This cohort is thus representative of the patient population eligible for treatment during that period and reflect outcomes for PLHIV starting the ART regimens recommended at that time.

\section{Conclusion}

In summary, while we found important associations with D-dimer levels and baseline characteristics as well as 
significant reductions in D-dimer following ART initiation, it should be noted that women and those with higher pre-treatment viral load appear to have persistently elevated levels which suggests continued risk for comorbidities including cardiovascular disease (CVD), and may be indicative of ongoing mortality risk [26]. Further research is needed to identify interventions that are effective at decreasing non-AIDS related morbidity and mortality among PLHIV on ART.

\begin{abstract}
Abbreviations
3TC: Lamivudine; ART: Antiretroviral therapy; ARTIC: Antiretroviral Therapy and Inflammatory and Coagulation Biomarkers; BMI: Body mass index; CDC: US Centers for Disease Control and Prevention; CUMC: Columbia University Medical Center; EFV: Efavirenz; HIV: Human immunodeficiency syndrome; IQR: Interquartile range; IL-6: Interleukin 6; IRB: Institutional review board; KEMRI: Kenya Medical Research Institute; NNRTI: Non-nucleoside reverse transcriptase inhibitor; $\mathrm{mL}$ : Milliliter; ng: Nanograms; NIH: National Institutes of Health; OAR: Office of AIDS Research; PLHIV: People living with HIV; TB: Tuberculosis; TDF: Tenofovir; VL: Viral load; WHO: World Health Organization
\end{abstract}

\section{Acknowledgements}

The authors would like to thank the study participants who made the study possible, the study staff and health facility personnel and administrators who provided care to the participants.

\section{Authors' contributions}

WME, MH, AZ, DC designed the study and oversaw collection of study data, CAT, WME, AZ, CH designed the analysis, CAT, CH conducted the analysis, and all contributed to manuscript writing and review. The author(s) read and approved the final manuscript.

\section{Funding}

Research reported in this paper is supported by the National Institutes of Health through the Office of AIDS Research under the terms of Award Number OAR 1B01NIAIDCU52002.

\section{Availability of data and materials}

Data from the ARTIC study are available upon request; please contact Allison Zerbe at ICAP at Columbia University, az2258@columbia.edu

\section{Ethics approval and consent to participate}

All participants provided written informed consent to participate in the ARTIC study. The ARTIC study was approved by the Kenya Medical Research Institute (KEMRI) and the Columbia University Medical Center (CUMC) Institutional Review Board (IRB).

\section{Consent for publication}

Not applicable.

\section{Competing interests}

The authors declare no competing interests.

\section{Author details}

'Department of Epidemiology and Biostatistics, CUNY Graduate School of Public Health and Health Policy, 55 W125th Street, Room 543, New York, NY 10027, USA. ${ }^{2}$ ICAP at Columbia University, Mailman School of Public Health, Columbia University, 22 W168th Street, New York, NY 10032, USA.

${ }^{3}$ Department of Epidemiology, Mailman School of Public Health, Columbia University, 722 W168th Street, New York, NY 10032, USA.

Received: 17 April 2020 Accepted: 30 June 2020

Published online: 14 July 2020

\section{References}

1. Baker JV, Sharma S, Grund B, et al. Systemic inflammation, coagulation, and clinical risk in the START trial. Open Forum Infect Dis. 2017;4(4):ofx262.
2. Boulware DR, Hullsiek KH, Puronen CE, et al. Higher levels of CRP, D-dimer, IL-6, and hyaluronic acid before initiation of antiretroviral therapy (ART) are associated with increased risk of AIDS or death. J Infect Dis. 2011;203(11): 1637-46.

3. Ledwaba L, Tavel JA, Khabo P, et al. Pre-ART levels of inflammation and coagulation markers are strong predictors of death in a south African cohort with advanced HIV disease. PLoS One. 2012;7(3):e24243.

4. Kuller LH, Tracy R, Belloso W, et al. Inflammatory and coagulation biomarkers and mortality in patients with HIV infection. PLoS Med. 2008; 5(10):e203.

5. Tenorio AR, Zheng Y, Bosch RJ, et al. Soluble markers of inflammation and coagulation but not T-cell activation predict non-AIDS-defining morbid events during suppressive antiretroviral treatment. J Infect Dis. 2014;210(8):1248-59.

6. Siedner MJ, Kim JH, Nakku RS, et al. Persistent immune activation and carotid atherosclerosis in HIV-infected Ugandans receiving antiretroviral therapy. J Infect Dis. 2016;213(3):370-8.

7. Lee S, Byakwaga H, Boum Y, et al. Immunologic pathways that predict mortality in HIV-infected Ugandans initiating antiretroviral therapy. J Infect Dis. 2017;215(8):1270-4.

8. Sullivan PS, Dworkin MS, Jones JL, Hooper WC. Epidemiology of thrombosis in HIV-infected individuals. The adult/adolescent spectrum of HIV disease project. AIDS. 2000;14(3):321-4.

9. Howard JFB, Rokx C, Smit C, et al. Incidence of a first venous thrombotic event in people with HIV in the Netherlands: a retrospective cohort study. Lancet HIV. 2019;6(3):e173-e81.

10. Levine AM, Vigen C, Gravink J, et al. Progressive prothrombotic state in women with advancing HIV disease. J Acquir Immune Defic Syndr. 2006; 42(5):572-7.

11. Funderburg NT. Markers of coagulation and inflammation often remain elevated in ART-treated HIV-infected patients. Curr Opin HIV AIDS. 2014;9(1): 80-6.

12. Carr A, Samaras K, Thorisdottir A, et al. Diagnosis, prediction, and natural course of HIV-1 protease-inhibitor-associated lipodystrophy, hyperlipidaemia, and diabetes mellitus: a cohort study. Lancet. 1999;353(9170):2093-9.

13. Friis-Moller $N$, Sabin CA, Weber $R$, et al. Combination antiretroviral therapy and the risk of myocardial infarction. N Engl J Med. 2003; 349(21):1993-2003.

14. Wada NI, Jacobson LP, Margolick JB, et al. The effect of HAART-induced HIV suppression on circulating markers of inflammation and immune activation. AIDS. 2015;29(4):463-71.

15. Baker JV, Neuhaus J, Duprez D, et al. Changes in inflammatory and coagulation biomarkers: a randomized comparison of immediate versus deferred antiretroviral therapy in patients with HIV infection. J Acquir Immune Defic Syndr. 2011;56(1):36-43.

16. Castillo-Mancilla JR, Morrow M, et al. Brief report: higher ART adherence is associated with lower systemic inflammation in treatment-naive Ugandans who achieve virologic suppression. J Acquir Immune Defic Syndr. 2018; 77(5):507-13.

17. Shivakoti $R$, Yang WT, Berendes $S$, et al. Persistently elevated C-reactive protein level in the first year of antiretroviral therapy, despite virologic suppression, is associated with HIV disease progression in resourceconstrained settings. J Infect Dis. 2016;213(7):1074-8.

18. Kenya National AIDS \& STI Contorl Program. Guidlines on use of antiretroviral drugs for treating and prevening HIV infection: rapid advice. Nairobi: KEMRI; 2014

19. Bounameaux H, de Moerloose P, Perrier A, Reber G. Plasma measurement of D-dimer as diagnostic aid in suspected venous thromboembolism: an overview. Thromb Haemost. 1994;71(1):1-6.

20. World Health Organization (WHO). Global health observatory visualization: indicator metadata registry. 2019. Available at: http://apps.who.int/gho/data/ node.wrapper.imr? $\mathrm{x}$-id=1

21. Kiefer EM, Hoover DR, Shi Q, et al. Longitudinal evaluation of markers of inflammation in HIV-positive and HIV-negative Rwandan women. HIV Med. 2018;19(10):734-44.

22. Borges AH, O'Connor UL, Phillips AN, et al. Factors associated with D-dimer levels in HIV-infected individuals. PLoS One. 2014;9(3):e90978.

23. Lee AJ, Fowkes GR, Lowe GD, Rumley A. Determinants of fibrin D-dimer in the Edinburgh artery study. Arterioscler Thromb Vasc Biol. 1995;15(8):1094-7.

24. Kabrhel C, Mark Courtney D, Camargo CA, et al. Factors associated with positive D-dimer results in patients evaluated for pulmonary embolism. Acad Emerg Med. 2010;17(6):589-97. 
25. Siedner MJ, Bwana MB, Asiimwe S, et al. Timing of antiretroviral therapy and systemic inflammation in sub-Saharan Africa: results from the META longitudinal cohort study. J Infect Dis. 2019;220(7):1172-7.

26. Kager LM, Blok DC, Lede IO, et al. Pulmonary tuberculosis induces a systemic hypercoagulable state. J Inf Secur. 2015;70(4):324-34.

27. Min W, Zi-Feng J, Jian-Lin X, Hao-Hui F. Role of the fibrinogen degradation products and $\mathrm{D}$-dimer in the differential diagnosis of pulmonary tuberculosis and community-acquired pneumonia. Clin Lab. 2018;64(1):135-40

28. Janssen S, Huson MAM, Osbak KK, et al. HIV infection rather than concurrent opportunistic infections drives most systemic procoagulant, vascular and damage responses - a prospective cohort study in central Africa. Antivir Ther. 2017;22:153-61.

29. Global tuberculosis report 2019. Geneva: World Health Organization; 2019. Available at: https:/apps.who.int/iris/bitstream/handle/10665/329368/ 9789241565714-eng.pdf?ua=1. Accessed 1 Mar 2020.

\section{Publisher's Note}

Springer Nature remains neutral with regard to jurisdictional claims in published maps and institutional affiliations.

Ready to submit your research? Choose BMC and benefit from:

- fast, convenient online submission

- thorough peer review by experienced researchers in your field

- rapid publication on acceptance

- support for research data, including large and complex data types

- gold Open Access which fosters wider collaboration and increased citations

- maximum visibility for your research: over $100 \mathrm{M}$ website views per year

At BMC, research is always in progress.

Learn more biomedcentral.com/submissions 